\title{
The Southern Regional Science Association and The Review of Regional Studies: History and Characteristics
}

\author{
Garey C. Durden and Hugh W. Knox*
}

\section{INTRODUCTION AND BACKGROUND}

This paper summarizes some characteristics of the Southern Regional Science Association (SRSA), attendance at yearly meetings of the SRSA, and the participation of individuals and institutions in presented paper sessions at those meetings. We also provide a detailed analysis of publishing performance, by individuals and institutions, in The Review of Regional Studies, which is the official publication of the SRSA.

Although reviews of associations such as the SRSA are relatively rare, there is some precedent, including the fine retrospective by Oliver Benson and Charles Bonjean (1970) on the Southwest Social Science Association and its publication, the Social Science Quarterly, for the period 1920-1970. Papers that have primarily analyzed publishing performance in a particular journal include those by Kau and Johnson (1983, Journal of Regional Science); Durden and Marlin (1990, Public Choice); Durden (1991, Social Science Quarterly); Shogren and Durden (1991, Journal of Environmental Economics and Management); and Marlin and Durden (1993, Journal of Economic Education).

This is a propitious time for such a paper: the SRSA is nearing the fortieth anniversary of its conception; yearly meetings, with paper presentations and discussions, are now in their thirtieth year; and The Review of Regional Studies is celebrating its thirtieth year of publication. The paper also provides the opportunity to recognize many of the people who have contributed to the success of the organization, primarily through participation in SRSA meetings and publication of papers in The Review.

\section{HISTORY OF THE SOUTHERN REGIONAL SCIENCE ASSOCIATION}

On March 27, 1962, Walter Isard visited the University of North Carolina at Chapel Hill to investigate interest in setting up a Southeastern Section of the Regional Science Association (the initial name of the SRSA). ${ }^{1}$ Lawrence Mann, then a young professor of planning at UNC-Chapel Hill, had earlier issued letters of invitation to potential members throughout the Southeast to attend an

\footnotetext{
*Professor of Economics, Appalachian State University, Boone, NC; and Associate Director for Regional Economics, Bureau of Economic Analysis, U.S. Department of Commerce, Washington, D.C. The views expressed in this paper are solely the author's and do not necessarily reflect the views of either the Bureau of Economic Analysis or the U.S. Department of Commerce.

1 The authors thank many members of the SRSA for sharing their records about, and reminiscences from, the early days of the SRSA. We thank Shirley Weiss, in particular, for detailed letters and notes from the earliest days of the SRSA.
} 
"Exploratory Organization Meeting." Four papers—by Walter Isard, Rutledge Vining, Lowell Ashby, and George Hall-were scheduled for that first meeting. Three were presented; the fourth, by Vining, was presented at a later meeting.

A second letter from Mann in October 1962 announced a formal organizational meeting to be held on November 9, 1962, in Atlanta, Georgia, where four more papers-by Isard, Vining, Joseph Airov, and John Friedmann--were presented, officers were elected, and a draft constitution was adopted. Those four papers were the first papers available from the nascent Southeastern Section of the RSA, at a cost of $\$ 1.00$ from the Center for Urban and Regional Studies in Chapel Hill.

A second annual meeting was called by Airov in Roanoke, Virginia, in November 1963 and featured discussants and, for the first time, an evening business meeting. At this meeting papers were presented by Donald Reeb, T.R. Lakshmanan, Mann, Ashby, Edgar Dunn, and John Wells, with discussion by Roger Burford and Allan Young.

We do not have any records indicating that further SRSA meetings took place during the remainder of the 1960s. However, there was intense regional science activity in the Southeast as elsewhere in the nation during this period. Major funding opportunities from the Area Redevelopment Administration and the Economic Development Administration (EDA), including the Title V commissions such as the Coastal Plains Regional Commission and the Appalachian Regional Commission, meshed with an unprecedented demand for regional science training and research from institutions such as the economic development districts of EDA and the many metropolitan planning agencies that grew up around transportation, water, housing, and environmental funding opportunities.

By the 1970s, southern regional scientists were once again trying to formalize an ongoing association and this time the effort bore lasting fruit. According to the SRSA Mission Statement, "The main objectives of the Association are the fostering and exchange of ideas and the promotion of studies focusing on the region including the utilization of tools, methods and theoretical frameworks specifically designed for regional analysis as well as concepts, procedures and analytical techniques of the various social and other sciences. These objectives are supported by the Association through the promotion of acquaintance and discussion among its members and with scholars in related fields, by the encouragement of publication of scholarly studies and by performing services to aid the advancement of its members and the field of regional science."2 This last sentence is the basis for yearly meetings and paper presentations, and for support of The Review of Regional Studies as the official publication of the SRSA.

Since its inception, the SRSA has been fortunate to have had a steady series of dedicated, resourceful, and accomplished individuals to serve as president of the organization. These are listed in Table 1, beginning with Robert T. Miki of the U.S. Department of Commerce (1971-1972) and continuing through John Rees from UNC-Greensboro (2000-2001). Where two institutions are listed in Table 1,

${ }^{2}$ From the SRSA Mission Statement, <www.gmu.edu/departments/tipp/policysite/srsa> 
the first is that with which the president was associated when elected and the second is the institution with which the individual is now affiliated.

TABLE 1

Presidents of the Southern Regional Science Association

\begin{tabular}{llc}
\hline Name & Institution & Year of Service \\
\hline Robert T. Miki & Department of Commerce & $1971-1972$ \\
William A. Schaffer & Georgia Tech & $1972-1973$ \\
Alan R. Winger & Fed. Home Loan Bank Cincinnati & $1973-1974$ \\
James M. Stepp & Clemson & $1974-1975$ \\
William H. Miernyk & West Virginia (Miernyk \& Associates) & $1975-1976$ \\
Monroe Newman & Penn State (Department of Commerce) & $1976-1977$ \\
Shirley F. Weiss & North Carolina & $1977-1978$ \\
Lowell D. Ashby & Department of Commerce & $1978-1979$ \\
Niles M. Hansen & Texas & $1979-1980$ \\
John H. Cumberland & Maryland & $1980-1981$ \\
Richard J. Olsen & Applied Business Research & $1981-1982$ \\
William J. Serow & Florida State & $1982-1983$ \\
Ray Grimes & Oklahoma State (Coopers and Lybrand) & $1983-1984$ \\
James C. Hite & Clemson & $1984-1985$ \\
Barry M. Moriarty & North Carolina & $1985-1986$ \\
Hugh W. Knox & Department of Commerce & $1986-1987$ \\
William R. Latham III & Delaware & $1987-1988$ \\
Joseph V. Cartwright & Department of Commerce & $1988-1989$ \\
David W. Rasmussen & Florida State & $1989-1990$ \\
Mark S. Henry & Clemson & $1990-1991$ \\
Andrew M. Isserman & West Virginia (Illinois) & $1991-1992$ \\
Thomas G. Johnson & Virginia Tech (Missouri) & $1992-1993$ \\
John R. Kort & Department of Commerce & $1993-1994$ \\
Edward T. Malecki & Florida & $1994-1995$ \\
Carol T. West & Florida & $1995-1996$ \\
David Mulkey & Florida & $1996-1997$ \\
David Barkley & Clemson & $1997-1998$ \\
G. Andrew Bernat, Jr. & Department of Commerce & $1998-1999$ \\
Judith Stallmann & Texas A\&M & $1999-2000$ \\
John Rees & UNC-Greensboro & $2000-2001$ \\
\hline
\end{tabular}

The duties of president include, as president-elect, supervision of preparations for the association's meetings for that year, including development of the program of paper presentations, and delivery of an address to the organization's members at the end of the presidential term. The text of the address is included in a subsequent issue of The Review of Regional Studies.

Periodically, individuals are selected to become Fellows of the SRSA. This honor is bestowed sparingly and is only conferred upon individuals who have made truly outstanding contributions to the field of regional science. Table 2 lists the 12 persons who have been honored as Fellows.

The first editors of The Review were Robert T. Miki of the U.S. Department of Commerce and Stanley E. Boyle of Virginia Tech. Personnel and financial support were provided by the Department of Economics and College of Business at Virginia Tech. Later, financial and other support was provided by the University of Alabama-Birmingham School of Business. (Although at one point, the proofs were held hostage by the printer in a labor dispute.) James C. Hite of Clemson 
University assumed editorship of The Review in 1979, and support, beginning in 1983, was provided by the Strom Thurmond Institute at Clemson. Editorship passed to Alan Schlottman and Henry Herzog at the University of Tennessee in Knoxville in 1990. In 1991, support efforts shifted from the Thurmond Institute to the College of Business Administration, University of Tennessee. William R. Latham III and Steven E. Hastings, both of the University of Delaware, are the current editors of The Review, and support efforts are provided by the College of Business and Economics at Delaware.

TABLE 2

Fellows of the Southern Regional Science Association

\begin{tabular}{ll}
\hline Name & Institution \\
\hline Walter Isard & Cornell \\
Edgar S. Dunn & Resources for the Future \\
William H. Miernyk & West Virginia \\
Lowell D. Ashby & U.S. Department of Commerce \\
Rutledge Vining & Virginia \\
F. Stuart Chapin, Jr. & North Carolina at Chapel Hill \\
Charles H. Leven & Washington U., St. Louis \\
William A. Schaffer & Georgia Tech \\
Niles M. Hansen & Texas at Austin \\
Brian J. Berry & Texas at Dallas \\
James C. Hite & Clemson \\
John H. Cumberland & Maryland \\
\hline
\end{tabular}

\section{MEMBERSHIP AND PARTICIPATION IN MEETINGS}

According to information supplied by Co-Executive Director Kevin McNamara of Purdue University, attendance at meetings of the SRSA for the last decade was as follows: 149 in 1991, 183 in 1992, 164 in 1993, 151 in 1994, 143 in 1995, 192 in 1996, 188 in 1997, 169 in 1998, and 178 in 1999, with an average of 169 members per year. A breakdown of membership by states compiled by the SRSA for 1987 and 1997 is given in Table 3. Table 3 shows that there were 352 members in 1987 and 308 in 1997, which is about twice the average paid membership. When these figures were compiled, member names were carried over for more than one year and thus persons were counted who were no longer paid members, exaggerating the true membership number. While this limits the use of the figures in Table 3, we feel that the relative magnitudes of membership among states would be much affected.

Table 3 also shows that, for the years reviewed, there has been at least one member of the SRSA from every state except Vermont. It is clear, however, that the majority of members come from the southern states, especially Florida, Georgia, South Carolina, North Carolina, and Tennessee, with lesser but substantial representation from Alabama, Kentucky, Louisiana, and West Virginia. Besides the Deep South, Texas is well represented. There is a large contingent from the various agencies in Washington D.C., consisting primarily of members of the Economic Research Service in the U.S. Department of Agriculture and the Bureau of 
Economic Analysis in the U.S. Department of Commerce. Memberships from D.C. fell off markedly in 1997 because more members from government agencies tended to list their state of residence (mostly Virginia or Maryland) rather than the District of Columbia.

TABLE 3

Membership in the Southern Regional Science Association, 1987 and 1997

\begin{tabular}{|c|c|c|}
\hline Name & 1987 & 1997 \\
\hline Alabama & 11 & 6 \\
\hline Arizona & 1 & 3 \\
\hline Arkansas & 3 & 2 \\
\hline California & 0 & 3 \\
\hline Colorado & 1 & 0 \\
\hline Connecticut & 1 & 0 \\
\hline Delaware & 6 & 5 \\
\hline Florida & 21 & 24 \\
\hline Georgia & 18 & 14 \\
\hline Hawaii & 1 & 0 \\
\hline Illinois & 9 & 8 \\
\hline Indiana & 5 & 4 \\
\hline Iowa & 0 & 2 \\
\hline Kansas & 1 & 3 \\
\hline Kentucky & 5 & 10 \\
\hline Louisiana & 11 & 5 \\
\hline Maryland & 7 & 7 \\
\hline Massachusetts & 5 & 3 \\
\hline Michigan & 3 & 3 \\
\hline Minnesota & 0 & 3 \\
\hline Mississippi & 9 & 5 \\
\hline Missouri & 4 & 5 \\
\hline Montana & 0 & 1 \\
\hline Nebraska & 7 & 2 \\
\hline Nevada & 1 & 4 \\
\hline New Jersey & 1 & 3 \\
\hline New Mexico & 0 & 1 \\
\hline New York & 6 & 5 \\
\hline North Carolina & 25 & 20 \\
\hline Ohio & 6 & 7 \\
\hline Oklahoma & 9 & 4 \\
\hline Oregon & 0 & 2 \\
\hline Pennsylvania & 6 & 15 \\
\hline Rhode Island & 1 & 0 \\
\hline South Carolina & 29 & 17 \\
\hline Tennessee & 31 & 10 \\
\hline Texas & 10 & 13 \\
\hline Vermont & 0 & 0 \\
\hline Virginia & 20 & 30 \\
\hline Washington D.C. & 31 & 19 \\
\hline Washington State & 0 & 2 \\
\hline West Virginia & 5 & 17 \\
\hline Wisconsin & 5 & 8 \\
\hline Wyoming & 1 & 5 \\
\hline Foreign & 7 & 8 \\
\hline Total & 352 & 308 \\
\hline
\end{tabular}

Source: Southern Regional Science Association Membership Directories, 1987 and 1997. 
A breakdown of SRSA membership by specialty fields is shown in Table 4 . Members typically list two or more specialties, so that totals are not meaningful. Consistently well-represented specialties include Applied Location Studies, Econometric Techniques, Impact and Location Modeling, Migration and Natural Resource Policy, Regional Development Policy, Growth Theory, Labor Market Analysis, Rural Development, and Taxation and Public Finance.

TABLE 4

Membership by Fields, 1987 and 1997

\begin{tabular}{|c|c|c|}
\hline Specialty & 1987 & 1997 \\
\hline Applied Location Studies & 22 & 19 \\
\hline Banking and Finance & 10 & 9 \\
\hline Capital Accumulation and Flow & 3 & 0 \\
\hline Central Place Theory & 2 & 1 \\
\hline City Planning & 4 & 6 \\
\hline Diffusion Studies & 1 & 0 \\
\hline Econometric Techniques & 36 & 14 \\
\hline Economic Demography & 10 & 14 \\
\hline Economic Geography & 23 & 0 \\
\hline Energy Policy & 13 & 3 \\
\hline Environmental Quality & 14 & 14 \\
\hline Gravity Models & 2 & 4 \\
\hline Human Capital and Manpower & 13 & 17 \\
\hline Impact Models & 38 & 35 \\
\hline Industrial Location, Development & 21 & 59 \\
\hline Input-Output Studies & 33 & 35 \\
\hline International Trade & 5 & 9 \\
\hline Interregional Trade & 4 & 0 \\
\hline Land Use and Prices & 13 & 6 \\
\hline Law/Police Administration & 2 & 5 \\
\hline Location Theory & 18 & 15 \\
\hline Marketing & 10 & 6 \\
\hline Migration & 21 & 17 \\
\hline Natural Resource Policy & 20 & 26 \\
\hline No Specialty Listed or other & 88 & 17 \\
\hline Pop Estimates and Projections & 14 & 5 \\
\hline Regional Development Policy & 51 & 63 \\
\hline Regional Forecasting, Modeling & 17 & 39 \\
\hline Regional Growth Theory & 24 & 30 \\
\hline Regional Labor Markets & 23 & 33 \\
\hline Regional Multipliers & 0 & 8 \\
\hline Regional Planning Theory & 8 & 3 \\
\hline Regional Statistics, Accounts & 14 & 21 \\
\hline Regional Governance & 3 & 2 \\
\hline Rural Development & 37 & 73 \\
\hline Social Welfare & 1 & 8 \\
\hline Spatial Markets and Prices & 10 & 8 \\
\hline Taxation and Public Finance & 26 & 33 \\
\hline Tests of Discrimination & 2 & 0 \\
\hline Tourism and Recreation & 14 & 14 \\
\hline Transportation & 12 & 6 \\
\hline Urban Redevelopment & 8 & 8 \\
\hline Urban Real Estate, Housing & 15 & 10 \\
\hline Utility Economics & 3 & 2 \\
\hline Water Resources Planning & 2 & 7 \\
\hline
\end{tabular}

Source: Southern Regional Science Association Membership Directories, 1987-1997. 
Table 5 lists the 190 individuals who have authored or coauthored two or more papers presented during one of the yearly SRSA meetings held during the period 1992-1999. The second data column of Table 5 shows the ranking of individuals according to the number of times his or her name has appeared on a presented paper (shown in the first data column). Tom Johnson, formerly at Virginia Tech and now at Missouri, has been represented more than any other individual at 16 times. Mark Henry of Clemson has been represented 13 times and Brian Cushing (West Virginia), Kingsley Haynes (George Mason), and Mark Partridge (St. Cloud State) have each been represented 12 times. Garey Durden (Appalachian State), Dan Rickman (Oklahoma State), Judy Stallmann (Texas A\&M), and Roger Stough (George Mason) are represented 11 times, and Steve Deller (Wisconsin), has authored or coauthored 10 presented papers. More than 150 others have authored or coauthored presented papers between two and nine times over the eight-year review period.

\section{TABLE 5}

Persons who have Authored or Coauthored Papers Presented During the Annual Meetings of the Southern Regional Science Association

\begin{tabular}{llcr}
\hline Name & Institution & $\begin{array}{c}\text { Number of } \\
\text { Appearances }\end{array}$ & Rank \\
\hline Alwang, Jeffrey & Virginia Tech & 5 & 40 \\
Ambargis, Zoe & U.S. Department of Commerce & 2 & 142 \\
Amey, Robert & Florida & 2 & 142 \\
Arena, Peter & George Mason & 4 & 61 \\
Azzoni, Carlos & U of San Paulo & 5 & 40 \\
Bagi, Faqir & U.S. Dept. of Agriculture & 4 & 61 \\
Bailey, Thomas & Kentucky & 2 & 142 \\
Banai, Reza & Memphis & 3 & 84 \\
Bao, Shuming & Clemson & 3 & 84 \\
Barkley, David & Clemson & 8 & 15 \\
Basu, Rathin & Ferrum College & 2 & 142 \\
Beck, Roger & Southern Illinois & 3 & 84 \\
Bergman, Edward & North Carolina & 2 & 142 \\
Bernat, G. Andrew & U.S. Department of Commerce & 6 & 27 \\
Bishop, John & East Carolina & 3 & 84 \\
Blalock, Gale & Evansville & 2 & 142 \\
Blanciforti, Laura & West Virginia & 2 & 142 \\
Boush, Carlton & Florida & 2 & 142 \\
Broder, Joseph & Georgia & 4 & 61 \\
Brooks, Kerry & Clemson & 3 & 84 \\
Broomhall, David & Purdue & 4 & 61 \\
Calhoun, Samuel & U.S. Dept. of Agriculture & 4 & 61 \\
Campbell, Harrison & UNC-Charlotte & 3 & 84 \\
Carter, George & Southern Mississippi & 8 & 16 \\
Charney, Alberta & Arizona & 2 & 142 \\
Chembezi, Duncan & Alabama A\&M & 2 & 142 \\
Chen, Kuan-Chow & Bowling Green St. & 3 & 61 \\
Chiles, Ted & Auburn & 4 & 142 \\
Chow, Victor & West Virginia & 2 & 142 \\
Clark, David & Marquette & 3 & 61 \\
Clark, Joy & Auburn & 2 & \\
Combs, Paul & Appalachian State & 2 & \\
Connaughton, John & UNC-Charlotte & 2 & \\
Conte, Michael & Baltimore & & \\
Cooke, Steve & Idaho & 2 & \\
\hline
\end{tabular}


TABLE 5 (continued)

Persons who have Authored or Coauthored Papers Presented During the Annual Meetings of the Southern Regional Science Association

\begin{tabular}{|c|c|c|c|}
\hline Name & Institution & $\begin{array}{c}\text { Number of } \\
\text { Appearances }\end{array}$ & Rank \\
\hline Courbois, J.P. & Appalachian State & 3 & 84 \\
\hline Cromartie, John & U.S. Dept. of Agriculture & 2 & 142 \\
\hline Cruz, Robert & Barry Uं & 2 & 142 \\
\hline Cushing, Brian & West Virginia & 12 & 3 \\
\hline Davis, Ann & Marist College & 2 & 142 \\
\hline Deepak, Sri Devi & Florida & 2 & 142 \\
\hline Deller, Steven & Wisconsin & 10 & 10 \\
\hline Dinc, Mustafa & George Mason & 6 & 27 \\
\hline Doeksen, Gerald & Oklahoma State & 3 & 84 \\
\hline Domazlicky, Bruce & SE Missouri State & 2 & 142 \\
\hline Draper, David & New Hampshire & 2 & 142 \\
\hline Duffy, Neal & SUNY-Plattsburgh & 2 & 142 \\
\hline Durden, Garey & Appalachian State & 11 & 6 \\
\hline Eff, Anthon & Middle Tennessee State & 9 & 11 \\
\hline Elledge, Barry & Appalachian State & 2 & 142 \\
\hline English, Donald B.K. & U.S. Dept. of Agriculture & 2 & 142 \\
\hline Fawson, Christopher & Utah State & 2 & 142 \\
\hline Feser, Edward & North Carolina & 3 & 84 \\
\hline Findeis, Jill & Penn State & 2 & 142 \\
\hline Fisher, Dennis & Texas A\&M & 2 & 142 \\
\hline Fletcher, Jerald & West Virginia & 3 & 84 \\
\hline Flaharty, Chuck & Missouri & 2 & 142 \\
\hline Formby, John & Alabama & 4 & 61 \\
\hline Fournier, Stephen & West Virginia & 2 & 142 \\
\hline Freshwater, David & TVA & 2 & 142 \\
\hline Fuller, Stephen & George Mason & 2 & 142 \\
\hline Gabe, Todd & Ohio State & 3 & 84 \\
\hline Gaynor, Patricia & Appalachian State & 7 & 23 \\
\hline Gibbs, Robert & U.S. Dept. of Agriculture & 5 & 40 \\
\hline Goetz, Stephen & Kentucky & 5 & 40 \\
\hline Goode, Frank & Penn State & 4 & 61 \\
\hline Goss, Earnest & Creighton & 8 & 16 \\
\hline Green, Gary & Georgia & 2 & 142 \\
\hline Guimaraes, Paulo & South Carolina & 2 & 142 \\
\hline Gunther, William & Alabama-Huntsville & 2 & 142 \\
\hline Guoqiang, Shen & East Carolina & 2 & 142 \\
\hline Halstead, John & New Hampshire & 3 & 84 \\
\hline Hamilton, Gregory & Arkansas-Little Rock & 7 & 23 \\
\hline Hammond, George & West Virginia & 4 & 61 \\
\hline Hamrick, Karen & U.S. Dept. of Agriculture & 3 & 84 \\
\hline Hansen, Niles & Texas & 4 & 61 \\
\hline Hanson, David & Duquesne & 2 & 142 \\
\hline Harris, Thomas & Nevada Reno & 2 & 142 \\
\hline Hastings, Steven & Delaware & 6 & 27 \\
\hline Haynes, Kingsley & George Mason & 12 & 3 \\
\hline Hefner, Frank & C of Charleston & 6 & 27 \\
\hline Henderson, David & Ohio State & 5 & 40 \\
\hline Henry, Mark & Clemson & 13 & 2 \\
\hline Hirasuna, Donald & Minn House of Rep & 2 & 142 \\
\hline Hite, James $C$. & Clemson & 6 & 27 \\
\hline Holland, David & Washington State & 3 & 84 \\
\hline Hoover, Dale & NC State & 2 & 142 \\
\hline House, Verne & Clemson & 3 & 84 \\
\hline Hsu, Wan-Ling & Penn State & 2 & 142 \\
\hline Hughes, David & Louisiana State & 9 & 11 \\
\hline
\end{tabular}


TABLE 5 (continued)

Persons who have Authored or Coauthored Papers Presented During the Annual Meetings of the Southern Regional Science Association

\begin{tabular}{|c|c|c|c|}
\hline Name & Institution & $\begin{array}{c}\text { Number of } \\
\text { Appearances }\end{array}$ & Rank \\
\hline Hunt, Gary & Maine & 2 & 142 \\
\hline Ihlanfeldt, Keith & Georgia State & 2 & 142 \\
\hline Isserman, Andrew & Illinois & 9 & 11 \\
\hline Jensen, Leif & Penn State & 3 & 84 \\
\hline Johnson, Kenneth & U.S. Department of Commerce & 3 & 84 \\
\hline Johnson, Kirk & George Mason & 2 & 142 \\
\hline Johnson, Thomas & Missouri & 16 & 1 \\
\hline Jones, Lonnie & Texas A\&M & 5 & 40 \\
\hline Karlsson, Charlie & Jonkoping Business School & 2 & 142 \\
\hline Keeler, Andrew & Georgia & 3 & 84 \\
\hline Keith, John & Utah State & 2 & 142 \\
\hline $\mathrm{Ke}$, Shanzi & North Carolina & 3 & 84 \\
\hline Kilkenny, Maureen & Iowa State & 5 & 40 \\
\hline Kleckley, James & Problem Solving Research & 2 & 142 \\
\hline Klier, Thomas & FRB-Chicago & 3 & 84 \\
\hline Knapp, Thomas & Penn State & 3 & 84 \\
\hline Kort, John & U.S. Department of Commerce & 3 & 8 \\
\hline Kowkowski, Mary & U.S. Department of Labor & 2 & 142 \\
\hline Kozlowski, Paul & Toledo & 4 & 61 \\
\hline Kranner, Edit & West Virginia & 2 & 142 \\
\hline Kraybill, David & Ohio State & 8 & 16 \\
\hline Kriesel, Warren & Georgia & 8 & 16 \\
\hline Krikelas, Andrew & FRB-Ätlanta & 3 & 84 \\
\hline Kulkarni, Rajendra & George Mason & 2 & 142 \\
\hline Lahr, Michael & Urban Policy Research & 2 & 142 \\
\hline Lall, Somik & George Mason & 5 & 40 \\
\hline Latham, William R. III & Delaware & 5 & 40 \\
\hline Lee, Daniel & Miss Insts of Higher Learning & 3 & 84 \\
\hline Lenze, David & Florida & 4 & 61 \\
\hline Levernier, William & Georgia Southern & 6 & 27 \\
\hline Liner, Gaines & UNC-Charlotte & 5 & 40 \\
\hline London, James & Clemson & 5 & 40 \\
\hline Loveridge, Scott & West Virginia & 3 & 84 \\
\hline Lugar, Michael & North Carolina & 4 & 61 \\
\hline Malecki, Edward & Florida & 8 & 16 \\
\hline Markley, Deborah & Purdue & 3 & 84 \\
\hline Marlin, Matthew & Duquesne & 3 & 84 \\
\hline McGranahan, David & U.S. Dept. of Agriculture & 3 & 84 \\
\hline McNamara, Kevin & Purdue & 9 & 11 \\
\hline Mia, Jian & Missouri & 3 & 84 \\
\hline Miller, Wayne & Arkansas & 3 & 84 \\
\hline Moriarty, Barry & North Carolina & 3 & 84 \\
\hline Moss, Charles & Florida & 3 & 84 \\
\hline Mulkey, David & Florida & 6 & 27 \\
\hline Nissan, Edward & Southern Miss & 8 & 16 \\
\hline Nord, Mark & Wisconsin & 3 & 84 \\
\hline Otto, Daniel & Iowa State & 3 & 84 \\
\hline Paelink, Jean & George Mason & 3 & 84 \\
\hline Partridge, Mark & St. Cloud State & 12 & 3 \\
\hline Phillips, Keith & FRB-Dallas & 3 & 84 \\
\hline Phipps, Tim & West Virginia & 3 & 84 \\
\hline Porterfield, Shirley & Washington U St Louis & 5 & 40 \\
\hline Rainey, Daniel & Clemson & 3 & 84 \\
\hline Raper, Michael & West Georgia & 5 & 40 \\
\hline Rasmussen, David & Florida State & 3 & 84 \\
\hline
\end{tabular}




\section{TABLE 5 (continued)}

Persons who have Authored or Coauthored Papers Presented During the Annual Meetings of the Southern Regional Science Association

\begin{tabular}{|c|c|c|c|}
\hline Name & Institution & $\begin{array}{l}\text { Number of } \\
\text { Appearances }\end{array}$ & Rank \\
\hline Reeder, Richard & U.S. Dept. of Agriculture & 6 & 27 \\
\hline Regan, Tom & South Carolina & 5 & 40 \\
\hline Renkow, Mitch & NC State & 3 & 84 \\
\hline Rephann, Terry & West Virginia & 5 & 40 \\
\hline Reynolds, John & Florida & 3 & 84 \\
\hline Rickman, Dan & Oklahoma State & 11 & 6 \\
\hline Robinson, Dennis & U.S. Army Corps of Engineers & 7 & 23 \\
\hline Rogers, Cynthia & Oklahoma State & 6 & 27 \\
\hline Schaffer, William & Georgia Tech & 6 & 27 \\
\hline Schantz, Radford & Maryland & 3 & 84 \\
\hline Schluter, Gerald & U.S. Dept. of Agriculture & 6 & 27 \\
\hline Schoening, Niles & Alabama-Huntsville & 3 & 84 \\
\hline Scott, James & Missouri & 4 & 61 \\
\hline Seidl, Andrew & Colorado St. U & 3 & 84 \\
\hline Serow, William & Florida State & 3 & 84 \\
\hline Shaffer, Ron & Wisconsin & 5 & 40 \\
\hline Siegel, Paul & Tennessee & 5 & 40 \\
\hline Silver, Stephen & The Citadel & 3 & 84 \\
\hline Smith, Eldon D. & Kentucky & 3 & 84 \\
\hline Smith, Stephen & Penn State & 8 & 16 \\
\hline Smith, Will & West Virginia & 3 & 84 \\
\hline Solocha, Andrew & Toledo & 3 & 84 \\
\hline Stabler, Jack & U of Saskatchewan & 3 & 84 \\
\hline Stallmann, Judith & Texas A\&M & 11 & 6 \\
\hline Stenberg, Peter & U.S. Dept. of Agriculture & 6 & 27 \\
\hline Stough, Roger & George Mason & 11 & 6 \\
\hline Sullivan, Patrick & U.S. Dept. of Agriculture & 3 & 84 \\
\hline Sutton, Terry & SE Missouri State & 3 & 84 \\
\hline Swenson, David & Iowa State & 4 & 61 \\
\hline Tanjuakio, Rudolfo & Delaware & 4 & 61 \\
\hline Tavernier, Edmund & Rutgers & 4 & 61 \\
\hline Taylor-West, Carol & Florida & 5 & 40 \\
\hline Temel, Tugrul & Rutgers & 4 & 61 \\
\hline Thompson, Eric & Kentucky & 6 & 27 \\
\hline Throckmorton, Bruce & Tennessee Tech & 4 & 61 \\
\hline Traxler, Johannes & North Carolina & 4 & 61 \\
\hline Trice, Mark & George Mason & 4 & 61 \\
\hline Vozikiz, George & The Citadel & 4 & 61 \\
\hline Walzer, Norman & Western Illinois & 3 & 84 \\
\hline Waters, Edward & Washington State & 3 & 84 \\
\hline White, Nancy & Bucknell U & 3 & 84 \\
\hline Wojan, Timothy & Kentucky & 5 & 40 \\
\hline Woodward, Douglas & South Carolina & 3 & 84 \\
\hline Zheng, Buhong & Alabama & 7 & 23 \\
\hline Zimet, David & Florida & 5 & 40 \\
\hline
\end{tabular}

Source: Programs Guides for the Annual Meetings of the Southern Regional Science Association, 1992-1999.

Presentation of papers by institution is summarized in Table 6. The first data column shows, from each institution, the number of persons who have authored or coauthored presented papers, and the third column shows how many times the institution's name was represented over the 1992-1999 period. For example, there were three different authors (coauthors) from the University of Alabama 
and that institution's name appeared 12 times. The second and fourth columns are the respective rankings. The number of times an institution is represented will be biased upward in many cases because there can be more than one author of a given paper. For example, the three representatives from Alabama may have singly authored four papers each or they may have jointly coauthored only four papers. In either case, the institution's name would appear in the program 12 times.

\section{TABLE 6}

Institutions of Persons Appearing on the Program of the Annual Meetings of the Southern Regional Science Association, 1992-1999

\begin{tabular}{|c|c|c|c|c|}
\hline Institution & $\begin{array}{c}\text { Persons } \\
\text { Appearing }\end{array}$ & Rank & $\begin{array}{c}\text { Total } \\
\text { Appearances }\end{array}$ & Rank \\
\hline Alabama & 3 & 45 & 12 & 36 \\
\hline Alabama A\&M & 4 & 32 & 5 & 32 \\
\hline Alabama-Huntsville & 3 & 45 & 6 & 36 \\
\hline Appalachian State & 6 & 26 & 28 & 22 \\
\hline Arizona & 2 & 63 & 4 & 54 \\
\hline Arkansas & 4 & 32 & 7 & 32 \\
\hline Arkansas-Little Rock & 1 & 79 & 7 & 71 \\
\hline Auburn & 3 & 45 & 10 & 47 \\
\hline Baltimore & 2 & 63 & 3 & 64 \\
\hline U.S. Census Bureau & 2 & 63 & 4 & 64 \\
\hline Central Florida & 4 & 32 & 5 & 32 \\
\hline Clemson & 17 & 9 & 53 & 9 \\
\hline College of Charleston & 2 & 63 & 7 & 64 \\
\hline Colorado & 3 & 45 & 3 & 47 \\
\hline Colorado State & 1 & 79 & 3 & 71 \\
\hline U.S. Army Corps of Engineers & 4 & 32 & 11 & 32 \\
\hline Creighton & 3 & 45 & 10 & 47 \\
\hline Delaware & 9 & 15 & 21 & 15 \\
\hline U.S. Department of Agriculture & 34 & 2 & 69 & 1 \\
\hline U.S. Department of Commerce & 18 & 7 & 23 & 7 \\
\hline Duquesne & 1 & 79 & 3 & 71 \\
\hline East Carolina & 2 & 63 & 5 & 64 \\
\hline Florida & 40 & 1 & 69 & 1 \\
\hline Florida International & 2 & 63 & 3 & 64 \\
\hline Florida State & 7 & 22 & 12 & 22 \\
\hline FRB-Atlanta & 1 & 79 & 3 & 71 \\
\hline FRB-Dallas & 2 & 63 & 4 & 64 \\
\hline George Mason & 26 & 4 & 68 & 3 \\
\hline Georgia & 26 & 4 & 5 & 5 \\
\hline Georgia Southern & 3 & 45 & 8 & 47 \\
\hline Georgia State & 3 & 45 & 5 & 47 \\
\hline Georgia Tech & 4 & 32 & 8 & 36 \\
\hline Idaho & 2 & 63 & 3 & 64 \\
\hline Iowa State & 7 & 22 & 16 & 22 \\
\hline Kentucky & 13 & 10 & 10 & 9 \\
\hline Louisiana State & 12 & 11 & 11 & 11 \\
\hline Maine & 2 & 63 & 3 & 71 \\
\hline Marist College & 2 & 63 & 3 & 71 \\
\hline Marquette & 1 & 79 & 3 & 71 \\
\hline Memphis & 3 & 45 & 6 & 47 \\
\hline Middle Tennessee State & 1 & 79 & 9 & 71 \\
\hline Minnesota & 3 & 45 & 3 & 47 \\
\hline Miss Insts of Higher Learning & 4 & 32 & 7 & 36 \\
\hline Missouri & 9 & 15 & 15 & 16 \\
\hline NC State & 8 & 18 & 11 & 18 \\
\hline
\end{tabular}


TABLE 6 (continued)

Institutions of Persons Appearing on the Program of the Annual Meetings of the Southern Regional Science Association, 1992-1999

\begin{tabular}{|c|c|c|c|c|}
\hline Institution & $\begin{array}{l}\text { Persons } \\
\text { Appearing }\end{array}$ & Rank & $\begin{array}{c}\text { Total } \\
\text { Appearances }\end{array}$ & Rank \\
\hline Nebraska & 7 & 22 & 7 & 22 \\
\hline Nevada Reno & 4 & 32 & 6 & 36 \\
\hline New Hampshire & 4 & 32 & 7 & 36 \\
\hline North Carolina & 12 & 11 & 13 & 12 \\
\hline Ohio State & 9 & 15 & 16 & 16 \\
\hline Oklahoma State & 18 & 7 & 7 & 8 \\
\hline Oregon State & 2 & 63 & 3 & 71 \\
\hline Penn State & 23 & 6 & 6 & 6 \\
\hline Purdue & 8 & 18 & 18 & 19 \\
\hline Rutgers & 5 & 27 & 11 & 27 \\
\hline SC State Government & 7 & 22 & 8 & 22 \\
\hline SE Missouri State & 4 & 32 & 7 & 36 \\
\hline South Carolina & 4 & 32 & 11 & 36 \\
\hline South Florida & 5 & 27 & 5 & 27 \\
\hline Southern Illinois & 2 & 63 & 5 & 71 \\
\hline Southern Mississippi & 3 & 45 & 17 & 54 \\
\hline SUNY-Plattsburgh & 2 & 63 & 3 & 71 \\
\hline Tennessee Tech & 1 & 79 & 4 & 71 \\
\hline Tennessee & 12 & 11 & 12 & 13 \\
\hline Texas & 5 & 27 & 8 & 27 \\
\hline Texas A\&M & 8 & 18 & 21 & 19 \\
\hline The Citadel & 4 & 32 & 10 & 36 \\
\hline Toledo & 2 & 63 & 7 & 71 \\
\hline TVA & 5 & 27 & 5 & 27 \\
\hline $\mathrm{U}$ of Groningen & 3 & 45 & 4 & 54 \\
\hline U of San Paulo & 2 & 63 & 6 & 71 \\
\hline U of Saskatchewan & 4 & 32 & 6 & 50 \\
\hline U of Strathclyde & 3 & 45 & 6 & 50 \\
\hline UNC Charlotte & 5 & 26 & 14 & 24 \\
\hline Utah State & 3 & 45 & 5 & 56 \\
\hline Virginia Employment Commission & 3 & 45 & 4 & 65 \\
\hline Virginia Tech & 12 & 12 & 18 & 20 \\
\hline Wageningen Agricultural U & 3 & 45 & 3 & 72 \\
\hline Washington State & 4 & 32 & 7 & 40 \\
\hline Washington U of St Louis & 2 & 63 & 5 & 56 \\
\hline West Georgia & 3 & 45 & 7 & 40 \\
\hline West Virginia & 34 & 2 & 76 & 1 \\
\hline Western Illinois & 1 & 79 & 3 & 72 \\
\hline Western Kentucky & 3 & 45 & 3 & 72 \\
\hline Winthrop & 4 & 45 & 4 & 65 \\
\hline
\end{tabular}

Source: Programs Guides for the Annual Meetings of the Southern Regional Science Association, 1992-1999.

The University of Florida has been represented in meeting sessions by 40 different persons, while the U.S. Department of Agriculture (primarily the Economic Research Service) has been represented by 34 individuals. There have been 32 different representatives from West Virginia, 26 from George Mason and Georgia, 23 from Penn State, 18 from the U.S. Department of Commerce (primarily the Bureau of Economic Analysis) and Oklahoma State, 17 from Clemson, 13 from Kentucky, and 12 each from Louisiana State, North Carolina, Tennessee, and Virginia Tech. More than 70 other institutions have been represented three or more times. 


\section{PUBLICATION PERFORMANCE IN THE REVIEW OF REGIONAL STUDIES}

The Review began publication of refereed articles with Volume 3, Number 1, 1972-73. The first two volumes were collections of papers presented during yearly meetings, and that information is excluded from this analysis. The tables used in the analyses to follow are all computed from the data on papers published from Volume 3, Number 1, through Volume 29, Number 2 (the Fall 1999 issue).

Table 7 provides a breakdown of published papers and pages by institutional classification as developed by the Carnegie Foundation for the Advancement of Teaching (1987). Categories are generally based on program type, i.e., the number of Ph.D.'s granted, if any; the amount of grant monies generated; etc. (A complete explanation of classifications may be found in Durden 1991, p. 182.) Table 7 shows that there have been 6,881 pages and 597 articles published in The Review since the journal was initiated. As with most journals, the greatest percentages of pages and papers have been published by individuals from Research 1 and Research 2 institutions, in this case, about half. Scholars from Comprehensive 1 and 2 schools have published about 12 percent of the papers and pages and Doctoral 1 and 2 schools a little more than 17 percent. Individuals from government agencies, the U.S. Departments of Commerce and Agriculture in particular, have produced about 10 percent of pages and 13 percent of papers published.

TABLE 7

Pages and Papers Published in The Review of Regional Studies According to Carnegie Foundation Classification

(numbers and percentages rounded)

\begin{tabular}{lrrrr}
\hline Carnegie & Pages & $\%$ & Pages & $\%$ \\
Classification & 2,138 & 31.1 & 170 & 28.5 \\
\hline Reseárch 1 & 1,170 & 17.0 & 112 & 18.8 \\
Research 2 & 821 & 11.9 & 71 & 12.0 \\
Comprehensive 1 & 698 & 10.1 & 67 & 7.2 \\
Doctoral 1 & 482 & 7.0 & 43 & 7.2 \\
Doctoral 2 & 27 & 0.4 & 2 & 0.3 \\
Comprehensive 2 & 18 & 0.3 & 2 & 13.1 \\
Liberal Arts & 682 & 9.9 & 78 & 5.2 \\
Government & 294 & 4.3 & 31 & 3.9 \\
Foreign & 471 & 6.8 & 21 & 100.0 \\
Other & 6,881 & 100.0 & 597 & \\
Totals & &
\end{tabular}

Source: The Reciew of Regional Studies, Volume 3, Number 1, through Volume 29, Number 2.

Table 8 shows pages and papers published by the state or country in which the author's institution is located (where location could be determined). Not surprisingly, the District of Columbia and Deep South states are dominant. D.C. is ranked first in pages and papers produced with about 7.5 percent. Florida is second in pages and papers; Georgia is fourth in pages, third in papers; North Carolina sixth in pages, fifth in papers; Tennessee is seventh in pages, sixth in papers; South Carolina is eighth in pages, seventh in papers; and Alabama is tenth in pages, ninth in papers. These states account for more than 30 percent of total production and, together with D.C., about 40 percent. Texas is also in the top ten 
(third in pages, fourth in papers) and two non-South states, Pennsylvania and Illinois, are ranked, respectively, fifth in pages, eighth in papers and ninth in pages, tenth in papers.

\section{TABLE 8}

Papers and Pages Published in The Review of Regional Studies by State and Country of Author's Institution

\begin{tabular}{|c|c|c|c|c|}
\hline State or Country & $\begin{array}{l}\text { Adjusted } \\
\text { Pages }\end{array}$ & Rank & $\begin{array}{l}\text { Adjusted } \\
\text { Papers }\end{array}$ & Rank \\
\hline Alabama & 205.67 & 10 & 20.75 & 9 \\
\hline Alaska & 14.50 & 49 & 2.00 & 40 \\
\hline Arizona & 92.00 & 24 & 7.50 & 25 \\
\hline Australia & 38.50 & 36 & 4.50 & 32 \\
\hline California & 91.50 & 26 & 6.25 & 27 \\
\hline Canada & 88.00 & 27 & 8.00 & 23 \\
\hline Colorado & 63.83 & 30 & 4.67 & 31 \\
\hline Connecticut & 31.00 & 39 & 3.00 & 35 \\
\hline Delaware & 60.00 & 32 & 6.17 & 28 \\
\hline Florida & 468.50 & 2 & 47.83 & 2 \\
\hline Georgia & 409.83 & 4 & 46.50 & 3 \\
\hline Germany & 26.00 & 41 & 1.00 & 49 \\
\hline Greece & 13.00 & 50 & 1.00 & 49 \\
\hline Hawaii & 12.00 & 52 & 1.00 & 49 \\
\hline Idaho & 32.20 & 38 & 1.80 & 42 \\
\hline Illinois & 221.83 & 9 & 18.83 & 10 \\
\hline India & 3.00 & 59 & 1.00 & 49 \\
\hline Indiana & 112.50 & 22 & 9.67 & 20 \\
\hline Iowa & 16.50 & 47 & 1.50 & 43 \\
\hline Ireland & 7.00 & 54 & 0.50 & 57 \\
\hline Israel & 31.00 & 39 & 2.00 & 40 \\
\hline Kansas & 117.00 & 20 & 9.67 & 20 \\
\hline Kentucky & 68.67 & 28 & 5.00 & 29 \\
\hline Louisiana & 119.00 & 19 & 10.17 & 19 \\
\hline Maryland & 61.00 & 31 & 5.00 & 29 \\
\hline Massachusetts & 18.33 & 46 & 2.33 & 39 \\
\hline Michigan & 58.00 & 33 & 3.00 & 35 \\
\hline Minnesota & 67.33 & 29 & 3.83 & 33 \\
\hline Mississippi & 113.33 & 21 & 10.83 & 17 \\
\hline Missouri & 130.00 & 17 & 10.50 & 18 \\
\hline Nebraska & 98.83 & 23 & 7.33 & 26 \\
\hline Nevada & 173.33 & 13 & 11.17 & 16 \\
\hline New Jersey & 2.50 & 61 & 0.50 & 57 \\
\hline New Mexico & 8.00 & 53 & 1.00 & 49 \\
\hline New York & 91.83 & 25 & 7.83 & 24 \\
\hline Nigeria & 7.00 & 54 & 1.00 & 49 \\
\hline North Carolina & 358.17 & 6 & 32.83 & 5 \\
\hline North Dakota & 23.67 & 42 & 1.33 & 47 \\
\hline Norway & 20.00 & 45 & 1.00 & 49 \\
\hline Ohio & 170.50 & 14 & 18.17 & 11 \\
\hline Oklahoma & 191.50 & 12 & 15.00 & 13 \\
\hline Oregon & 5.00 & 58 & 0.50 & 57 \\
\hline Pennsylvania & 371.00 & 5 & 25.75 & 8 \\
\hline Philippines & 3.00 & 59 & 0.50 & 57 \\
\hline Puerto Rico & 13.00 & 50 & 1.50 & 43 \\
\hline Rhode Island & 35.00 & 37 & 1.50 & 43 \\
\hline South Carolina & 304.58 & 8 & 27.17 & 7 \\
\hline Tennessee & 356.00 & 7 & 29.08 & 6 \\
\hline Texas & 418.25 & 3 & 35.75 & 4 \\
\hline United Kingdom & 7.00 & 54 & 1.00 & 49 \\
\hline
\end{tabular}




\section{TABLE 8 (continued)}

Papers and Pages Published in The Review of Regional Studies by State and Country of Author's Institution

\begin{tabular}{lccrc}
\hline State or Country & $\begin{array}{c}\text { Adjusted } \\
\text { Pages }\end{array}$ & Rank & $\begin{array}{c}\text { Adjusted } \\
\text { Papers }\end{array}$ & Rank \\
\hline Utah & 22.33 & 43 & 1.33 & 47 \\
Vermont & 7.00 & 54 & 0.50 & 57 \\
Virginia & 147.33 & 16 & 13.33 & 14 \\
Washington & 16.00 & 48 & 2.50 & 38 \\
Washington D.C. & 522.30 & 1 & 50.78 & 1 \\
West Virginia & 125.17 & 18 & 9.67 & 20 \\
Wisconsin & 166.17 & 15 & 12.50 & 15 \\
Wyoming & 46.83 & 34 & 3.33 & 34 \\
\hline
\end{tabular}

Source: The Review of Regional Studies, Volume 3, Number 1, through Volume 29, Number 2.

TABLE 9

Papers and Pages Published in The Review of Regional Studies by Institution of Authors

\begin{tabular}{|c|c|c|c|c|}
\hline Institution & $\begin{array}{c}\text { Adjusted } \\
\text { Pages }\end{array}$ & Rank & $\begin{array}{c}\text { Adjusted } \\
\text { Papers }\end{array}$ & Rank \\
\hline Alabama (9) & 70.0 & 24 & 7.5 & 19 \\
\hline Alabama A\&M (2) & 7.5 & 130 & 1.0 & 104 \\
\hline Alabama Birmingham (3) & 14.0 & 101 & 2.3 & 65 \\
\hline Alabama Huntsville (4) & 15.8 & 97 & 1.8 & 80 \\
\hline American (2) & 12.0 & 111 & 2.0 & 66 \\
\hline Appalachian State (9) & 43.0 & 43 & 4.0 & 34 \\
\hline Arizona State West (3) & 20.5 & 78 & 1.5 & 86 \\
\hline Arkansas (4) & 27.0 & 62 & 3.0 & 45 \\
\hline Armstrong State (14) & 68.8 & 25 & 11.3 & 9 \\
\hline Atlanta (2) & 16.0 & 94 & 1.0 & 104 \\
\hline Auburn (3) & 33.5 & 52 & 2.0 & 66 \\
\hline Auburn Montgomery (3) & 18.0 & 85 & 1.3 & 100 \\
\hline Austin Peay (3) & 19.0 & 84 & 2.0 & 66 \\
\hline Australian Nat U (2) & 21.0 & 77 & 2.0 & 66 \\
\hline Ball State (2) & 8.0 & 128 & 1.0 & 104 \\
\hline Brown (2) & 35.0 & 49 & 1.5 & 86 \\
\hline Cal Riverside (2) & 1.5 & 143 & 0.8 & 132 \\
\hline Census Bureau (5) & 59.0 & 32 & 4.5 & 32 \\
\hline Central Florida (5) & 21.5 & 76 & 2.5 & 55 \\
\hline Clarion (2) & 14.0 & 101 & 1.5 & 86 \\
\hline Clemson (29) & 165.6 & 4 & 16.5 & 3 \\
\hline Colorado (6) & 49.8 & 36 & 3.7 & 39 \\
\hline Columbus Lab of Battle (4) & 16.0 & 94 & 1.0 & 104 \\
\hline Connecticut (3) & 24.3 & 67 & 1.8 & 80 \\
\hline Cornell (2) & 16.7 & 91 & 0.8 & 128 \\
\hline Creighton (5) & 47.3 & 38 & 3.3 & 43 \\
\hline Deakin U (3) & 13.5 & 106 & 1.5 & 86 \\
\hline Delaware (10) & 60.0 & 29 & 6.2 & 22 \\
\hline U.S. Dept. of Agriculture (21) & 149.8 & 6 & 12.5 & 5 \\
\hline U.S. Dept. of Commerce (10) & 77.5 & 18 & 9.0 & 15 \\
\hline U.S. Dept. of Defense (2) & 11.5 & 115 & 1.5 & 86 \\
\hline U.S. Dept. of Energy (6) & 59.5 & 31 & 5.5 & 24 \\
\hline East Carolina (3) & 18.0 & 85 & 2.0 & 66 \\
\hline East Texas State (2) & 9.7 & 122 & 0.8 & 128 \\
\hline Federal Reserve St Louis (2) & 11.0 & 117 & 1.0 & 104 \\
\hline Federal Reserve Dallas (2) & 9.0 & 123 & 1.0 & 104 \\
\hline FHLB Atlanta (4) & 31.0 & 57 & 2.5 & 55 \\
\hline Florida (22) & 138.3 & 10 & 12.2 & 6 \\
\hline
\end{tabular}


TABLE 9 (continued)

Papers and Pages Published in The Review of Regional Studies by Institution of Authors

\begin{tabular}{|c|c|c|c|c|}
\hline Institution & $\begin{array}{c}\text { Adjusted } \\
\text { Pages }\end{array}$ & Rank & $\begin{array}{c}\text { Adjusted } \\
\text { Papers }\end{array}$ & Rank \\
\hline Florida Atlantic (6) & 46.3 & 39 & 3.8 & 37 \\
\hline Florida State $(40)$ & 210.8 & 1 & 24.8 & 1 \\
\hline George Mason (3) & 17.2 & 88 & 1.3 & 100 \\
\hline George Washington (2) & 20.0 & 80 & 1.5 & 86 \\
\hline Georgia (32) & 148.3 & 7 & 17.3 & 2 \\
\hline Georgia Southern (2) & 23.3 & 70 & 1.3 & 100 \\
\hline Georgia State $(7)$ & 59.7 & 30 & 4.8 & 31 \\
\hline Georgia Tech $(7)$ & 39.0 & 46 & 5.5 & 24 \\
\hline Gov's Office Puerto Rico (2) & 9.0 & 123 & 1.0 & 104 \\
\hline Hawaii Honolulu (2) & 11.0 & 117 & 1.0 & 104 \\
\hline Idaho (6) & 32.2 & 54 & 1.8 & 80 \\
\hline Illinois (5) & 37.0 & 47 & 3.5 & 40 \\
\hline Illinois Chicago (7) & 45.0 & 40 & 3.0 & 45 \\
\hline Illinois State (4) & 18.0 & 85 & 3.5 & 40 \\
\hline Indiana $(7)$ & 44.7 & 41 & 3.8 & 37 \\
\hline Indiana Purdue Ft. Wayne (2) & 20.0 & 80 & 1.5 & 86 \\
\hline Iowa State (2) & 16.5 & 93 & 1.5 & 86 \\
\hline Kansas State (11) & 86.0 & 16 & 7.2 & 20 \\
\hline Kent State (2) & 6.0 & 134 & 2.0 & 66 \\
\hline Kentucky (9) & 73.3 & 22 & 5.2 & 28 \\
\hline Louisiana State (6) & 41.0 & 44 & 4.0 & 34 \\
\hline Marquette (5) & 48.0 & 37 & 3.0 & 45 \\
\hline Maryland (8) & 55.5 & 35 & 4.2 & 33 \\
\hline Massachusetts-Amherst (4) & 21.7 & 75 & 2.7 & 53 \\
\hline McNeese State (2) & 4.0 & 138 & 0.7 & 133 \\
\hline Memphis State (3) & 24.5 & 66 & 2.5 & 55 \\
\hline Middle Tenn State (4) & 43.7 & 42 & 2.7 & 53 \\
\hline Mississippi State (3) & 37.0 & 47 & 3.0 & 45 \\
\hline Missouri (15) & 95.8 & 15 & 9.8 & 15 \\
\hline Montana (2) & 7.0 & 131 & 0.5 & 135 \\
\hline NC State (2) & 20.0 & 80 & 1.5 & 86 \\
\hline Nebraska (3) & 31.5 & 56 & 2.5 & 55 \\
\hline Nevada Las Vegas (6) & 35.0 & 49 & 2.5 & 55 \\
\hline Nevada Reno (26) & 179.3 & 3 & 10.7 & 11 \\
\hline North Carolina-Chapel Hill (20) & 182.2 & 2 & 15.3 & 4 \\
\hline North Dakota St (2) & 14.7 & 100 & 0.8 & 128 \\
\hline North Texas (2) & 12.0 & 111 & 1.0 & 104 \\
\hline Northern Arizona (4) & 33.0 & 53 & 2.5 & 55 \\
\hline Norwegian U of Sci \& Tech (2) & 20.0 & 80 & 1.0 & 104 \\
\hline Oak Ridge National Labs (8) & 68.5 & 26 & 5.0 & 29 \\
\hline Oakland (2) & 22.0 & 73 & 1.0 & 104 \\
\hline Occidental College (2) & 32.0 & 55 & 1.0 & 104 \\
\hline Ohio (4) & 25.0 & 63 & 2.5 & 55 \\
\hline Ohio State (5) & 28.3 & 61 & 3.3 & 43 \\
\hline Oklahoma (5) & 34.0 & 51 & 3.0 & 45 \\
\hline Oklahoma State (19) & 157.5 & 5 & 12.0 & 7 \\
\hline Penn (5) & 30.5 & 59 & 3.0 & 45 \\
\hline Penn St Erie (2) & 22.0 & 73 & 0.7 & 133 \\
\hline Penn State (18) & 140.8 & 8 & 9.9 & 13 \\
\hline Pittsburgh (8) & 107.0 & 14 & 6.2 & 22 \\
\hline Rice (5) & 67.0 & 27 & 5.0 & 29 \\
\hline San Jose State (2) & 14.0 & 101 & 1.0 & 104 \\
\hline SE Louisiana State (2) & 17.0 & 89 & 1.5 & 86 \\
\hline SE Missouri State (5) & 57.0 & 33 & 3.0 & 45 \\
\hline South Alabama (5) & 24.0 & 68 & 3.5 & 40 \\
\hline
\end{tabular}


TABLE 9 (continued)

Papers and Pages Published in The Review of Regional Studies by Institution of Authors

\begin{tabular}{|c|c|c|c|c|}
\hline Institution & $\begin{array}{l}\text { Adjusted } \\
\text { Pages }\end{array}$ & Rank & $\begin{array}{c}\text { Adjusted } \\
\text { Papers }\end{array}$ & Rank \\
\hline South Carolina (11) & 94.0 & 15 & 7.2 & 20 \\
\hline South Florida (2) & 14.0 & 101 & 1.0 & 104 \\
\hline Southern IIl (8) & 73.5 & 21 & 5.5 & 24 \\
\hline Southern Methodist (3) & 13.0 & 107 & 2.0 & 66 \\
\hline Southern Mississippi (12) & 76.3 & 19 & 7.8 & 17 \\
\hline Southwestern Louisiana (2) & 13.0 & 107 & 1.5 & 86 \\
\hline St Cloud State (5) & 61.3 & 28 & 2.8 & 52 \\
\hline SUNY-Plattsburgh (2) & 16.0 & 94 & 2.0 & 66 \\
\hline Tel Hai College (3) & 22.3 & 72 & 1.3 & 100 \\
\hline Tenn Valley Authority (3) & 23.3 & 70 & 1.8 & 80 \\
\hline Tennessee (25) & 140.5 & 9 & 11.8 & 8 \\
\hline Tennessee Chattanooga (3) & 20.5 & 78 & 1.5 & 86 \\
\hline Texas (14) & 129.3 & 12 & 11.3 & 9 \\
\hline Texas Arlington (3) & 30.0 & 60 & 2.5 & 55 \\
\hline Texas A\&M (12) & 70.4 & 23 & 5.4 & 27 \\
\hline Texas-Dallas (2) & 24.0 & 68 & 2.0 & 66 \\
\hline The Urban Institute (4) & 23.0 & 71 & 2.0 & 66 \\
\hline U of British Columbia (2) & 15.5 & 98 & 1.5 & 86 \\
\hline U of Nottingham (2) & 7.0 & 131 & 1.0 & 104 \\
\hline U of Saskatchewan (5) & 41.0 & 44 & 2.3 & 64 \\
\hline U of Waterloo (4) & 25.0 & 63 & 2.0 & 66 \\
\hline UCLA (2) & 14.0 & 101 & 1.0 & 104 \\
\hline UNC-Charlotte (6) & 56.0 & 34 & 4.0 & 34 \\
\hline UNC-Greensboro (3) & 9.0 & 123 & 2.0 & 66 \\
\hline UNC-Wilmington (4) & 11.0 & 117 & 2.0 & 66 \\
\hline Union Pacific Railroad (2) & 17.0 & 89 & 1.0 & 104 \\
\hline Universidad Do Porto (2) & 13.0 & 107 & 0.8 & 128 \\
\hline Upjohn Institute (2) & 8.0 & 128 & 1.5 & 86 \\
\hline Valdosta State (4) & 15.0 & 99 & 2.0 & 66 \\
\hline Virginia (3) & 11.3 & 116 & 1.8 & 80 \\
\hline Virginia Tech (4) & 16.7 & 91 & 1.7 & 80 \\
\hline West Virginia (15) & 129.5 & 11 & 10.0 & 12 \\
\hline Wichita State (3) & 31.0 & 57 & 2.5 & 55 \\
\hline William and Mary (22) & 25.0 & 63 & 2.0 & 66 \\
\hline Winthrop (2) & 12.0 & 111 & 1.0 & 104 \\
\hline Wisconsin (20) & 124.2 & 13 & 9.2 & 14 \\
\hline Wright State (13) & 74.5 & 20 & 7.7 & 18 \\
\hline
\end{tabular}

Source: The Review of Regional Studies, Volume 3, Number 1, through Volume 29, Number 2.

Table 9 ranks the institutions of authors and coauthors according to total pages and papers published. The number in parentheses next to the institution name is the number of persons who have authored or coauthored papers and an institution must have had two or more such authors or coauthors to be listed. Data columns 1 and 3 show "Adjusted Pages" and "Adjusted Papers," meaning that pages and papers are adjusted for coauthors. Specifically, for a given paper, pages per author are (total pages/number of authors) and each author is credited with $1 / \mathrm{n}$ papers, where $\mathrm{n}$ is the number of authors. If a 20 -page paper has one author, that person is credited with 20 pages and one paper. If there are two coauthors, each receives credit for 10 pages and 0.5 papers. 
Florida State has had 40 different persons publish in The Review and ranks (all figures rounded) first both in papers (25) and pages (211) produced, followed by North Carolina, which is ranked second in pages (182), fourth in papers (15, with 20 persons contributing). Interestingly, the University of Nevada at Reno is third in pages produced (179), eleventh in the number of papers (11, with 26 contributors). Clemson ranks fourth in pages (166) and third in papers (17, with 29 contributors), followed by Oklahoma State (fifth in pages, 158, seventh in papers, 12, with 19 contributors), the U.S. Department of Agriculture (sixth in pages, 150, fifth in papers, 13, with 21 contributors), Georgia (seventh in pages, 148, second in papers, 17, with 32 contributors), Penn State (eighth in pages, 141, thirteenth in papers, 10, with 18 contributors), Tennessee (ninth in pages, 141, eighth in papers, 12 , with 25 contributors), and Florida (tenth in pages, 138, sixth in papers, 12, with 22 contributors). Among those in the top 10 in papers but not pages are Texas (twelfth in pages, 129, ninth in papers, 11, with 14 contributors) and Armstrong State (twenty-fifth in pages, 69 , but ninth in papers, 11 , with 14 contributors).

Table 10 shows a breakdown of papers and pages published by degree and/or type of discipline. Economics dominates, accounting for about 70 percent of the contributions. Agricultural Economics accounts for another 12 percent and Geographers published about 3 percent of pages and papers. No other discipline accounted for as much as 2 percent of the totals.

\section{TABLE 10}

Contributions by Degree Discipline of Authors

\begin{tabular}{lrrrr}
\hline Institution & $\begin{array}{c}\text { Adjusted } \\
\text { Pages }\end{array}$ & Rank & $\begin{array}{c}\text { Adjusted } \\
\text { Papers }\end{array}$ & Rank \\
\hline Economics & 4,816 & 69.8 & 418 & 70.0 \\
Agricultural Economics & 856 & 12.3 & 74 & 12.4 \\
Geography & 200 & 2.8 & 15 & 2.5 \\
Business/Finance & 128 & 1.8 & 11 & 1.8 \\
Poli Sci/Publ Admin/Gov't & 110 & 1.6 & 6 & 1.1 \\
Sociology & 85 & 1.2 & 5 & 1.0 \\
Planning & 80 & 1.2 & 5 & 1.0 \\
Real Estate/Urban Economics & 72 & 1.0 & 7 & 1.2 \\
Other & 800 & 11.5 & 56 & 9.3 \\
\hline
\end{tabular}

Source: The Review of Regional Studies, Volume 3, Number 1, through Volume 29, Number 2.

Table 11 lists individual contributors to The Review and notes the institution of each. To be listed, an individual must have been an author or coauthor of two or more papers, and there have been more than 150 such persons. Data columns 1 and 3 show papers and pages adjusted for coauthors, and data columns 2 and 4 again show rankings. Among the top ten in pages (ties included) are Niles Hansen of Texas (first in pages, 90, second in papers, 7.5); William Serow of Florida State (second in pages, 71, third in papers, 6.8); Orley Amos of Oklahoma State (third in pages, 66, fourth in papers, 6.3); Richard Cebula of Armstrong State (fourth in pages, 64, first in papers, 11); Mark Partridge of St. Cloud State (fifth in pages, 61, nineteenth in papers, 2.8); David Houston of Pittsburgh (sixth in pages, 56 , fourteenth in papers, 3); Edward Miller of Rice (seventh in pages, 55, eighth in 
papers, 4); Frederick Gale of the U.S. Department of Agriculture (eighth in pages, 53, fourteenth in papers, 3); Noel Uri of the U.S. Department of Energy (ninth in pages, 50, eighth in papers, 4); and Barry Moriarty of North Carolina (tenth in pages, 48 , fourteenth in papers, 3). Among the top ten in papers but not pages are David Mulkey of Florida (fifth in papers, 4.7, eleventh in pages, 45); Michael Babcock of Kansas State (sixth in papers, 4.5, thirteenth in pages, 44); Tom Johnson of Missouri (seventh in papers, 4.3, twelfth in pages, 45); and David Rasmussen of Florida State (eighth in papers, 4, thirty-third in pages, 27).

\section{TABLE 11}

Pages and Papers Published in The Review of Regional Studies by Author and Institution

\begin{tabular}{|c|c|c|c|c|c|}
\hline Author & Institution & $\begin{array}{l}\text { Idjusted } \\
\text { Pages }\end{array}$ & Rank & $\begin{array}{l}\text { Adjusted } \\
\text { Papers }\end{array}$ & Rank \\
\hline Amos, Orley M., Jr. & Oklahoma State (7) & 66.3 & 3 & 6.3 & 4 \\
\hline Andrikopoulos, Andreas A. & U of Waterloo (2) & 19.7 & 64 & 1.3 & 93 \\
\hline Ashby, Lowell D. & U.S. Dept. Commerce (3) & 22.0 & 45 & 3.0 & 14 \\
\hline Babcock, Michael W. & Kansas State (5) & 44.0 & 13 & 4.5 & 6 \\
\hline Barkley, David L. & Clemson (3) & 27.5 & 30 & 1.8 & 53 \\
\hline Beck, Roger J. & Southern Illinois (3) & 10.5 & 112 & 1.5 & 61 \\
\hline Benson, Bruce L. & Florida State (4) & 30.0 & 26 & 2.8 & 19 \\
\hline Bergstrom, Jon & Georgia (2) & 6.8 & 147 & 0.8 & 131 \\
\hline Bernat, G. Andrew, Jr. & U.S. Dept. Commerce (2) & 15.0 & 88 & 2.0 & 31 \\
\hline Blair, John P. & Wright State (3) & 15.5 & 85 & 1.7 & 60 \\
\hline Bohm, Robert A. & Tennessee (4) & 14.5 & 90 & 1.5 & 61 \\
\hline Braschler, Curtis & Missouri (2) & 8.0 & 133 & 0.8 & 131 \\
\hline Brucker, Sharon M. & Delaware (2) & 7.0 & 142 & 0.8 & 131 \\
\hline Brueggeman, William B. & Ohio State (2) & 12.0 & 100 & 2.0 & 31 \\
\hline Cahill, Edward E. & Tennessee (2) & 13.5 & 93 & 1.0 & 109 \\
\hline Campbell, Charles A. & Mississippi State (2) & 22.0 & 45 & 2.0 & 31 \\
\hline Carter, George H. & Southern Mississippi (2) & 12.0 & 100 & 1.5 & 61 \\
\hline Cartwright, Joseph V. & U.S. Dept. Defense (2) & 11.5 & 106 & 1.5 & 61 \\
\hline Caveny, Regina & Southern Mississippi (2) & 2.5 & 157 & 1.0 & 109 \\
\hline Cebula, Richard J. & Armstrong State (13) & 63.5 & 4 & 11.0 & 1 \\
\hline Centner, Terence J. & Georgia (2) & 7.0 & 142 & 0.8 & 131 \\
\hline Chang, Semoon & South Alabama (2) & 13.0 & 95 & 1.5 & 61 \\
\hline Clark, David E. & Marquette (2) & 23.0 & 39 & 1.0 & 109 \\
\hline Coelen, Stephen P. & Massachusetts Amherst (2) & 2) 9.3 & 123 & 1.3 & 93 \\
\hline Connaughton, John E. & UNC-Charlotte (2) & 9.5 & 121 & 1.0 & 109 \\
\hline Connaughton, Kent & U.S. Dept. Agric (2) & 7.3 & 141 & 0.5 & 156 \\
\hline Crane, Laurence M. & North Dakota State (2) & 14.7 & 89 & 0.8 & 131 \\
\hline Cumberland, John H. & Maryland (5) & 34.5 & 22 & 2.8 & 19 \\
\hline Davis, H. Craig & U of British Columbia (2) & 15.5 & 85 & 1.5 & 61 \\
\hline Deaton, Brady J. & Virginia Tech (2) & 6.3 & 149 & 0.8 & 131 \\
\hline Deller, Steven C. & Wisconsin (3) & 21.8 & 52 & 1.2 & 104 \\
\hline Deman, Suresh & Australian Nat U (2) & 21.0 & 56 & 2.0 & 31 \\
\hline Dillman, B.L. & Clemson (2) & 11.0 & 109 & 1.0 & 109 \\
\hline Domazlicky, Bruce R. & SE Missouri State (2) & 22.5 & 43 & 1.5 & 61 \\
\hline Duffy, Neal E. & SUNY-Plattsburgh (2) & 16.0 & 83 & 2.0 & 31 \\
\hline Durden, Garey C. & Appalachian State (5) & 26.7 & 32 & 2.3 & 28 \\
\hline Ebai, George & Nevada Reno (2) & 21.0 & 56 & 0.8 & 131 \\
\hline Eff, E. Anthon & Middle Tenn State (2) & 37.0 & 19 & 2.0 & 31 \\
\hline Ellerbrock, Michael J. & East Texas State (2) & 9.7 & 119 & 0.8 & 131 \\
\hline Emerson, M. Jarvin & Kansas State (3) & 18.5 & 73 & 1.2 & 104 \\
\hline Engels, Richard A. & U.S. Census Bureau (2) & 23.0 & 39 & 2.0 & 31 \\
\hline Epperson, James & Georgia (2) & 9.8 & 118 & 0.8 & 131 \\
\hline Erickson, Rodney A. & Penn State (2) & 22.0 & 45 & 1.5 & 61 \\
\hline Ervin, Osbin L. & Southern Illinois (2) & 30.0 & 26 & 2.0 & 31 \\
\hline
\end{tabular}




\section{TABLE 11}

Pages and Papers Published in The Review of Regional Studies by Author and Institution

\begin{tabular}{|c|c|c|c|c|c|}
\hline Author & Institution & $\begin{array}{l}\text { Idjusted } \\
\text { Pages }\end{array}$ & Rank & $\begin{array}{l}\text { Adjusted } \\
\text { Papers }\end{array}$ & Rank \\
\hline Farrell, Claude & UNC-Wilmington (2) & 5.5 & 155 & 1.0 & 109 \\
\hline Fichtenbaum, Rudy & Wright State (3) & 18.0 & 74 & 1.5 & 61 \\
\hline Floyd, Charles F. & Georgia (3) & 10.0 & 116 & 1.5 & 61 \\
\hline Fournier, Gary M. & Florida State (2) & 6.3 & 149 & 0.8 & 131 \\
\hline Fritz, Richard G. & Fed Home Loan Bank (4) & 31.0 & 24 & 2.5 & 25 \\
\hline Fuller, Stephen W. & Texas A\&M (2) & 15.7 & 84 & 1.3 & 93 \\
\hline Gale, H. Frederick & U.S. Dept. Agric. (3) & 53.0 & 8 & 3.0 & 14 \\
\hline Gaynor, Patricia E. & Appalachian State (2) & 9.7 & 119 & 0.8 & 131 \\
\hline Gerking, Shelby D. & Wyoming (2) & 21.0 & 56 & 1.0 & 109 \\
\hline Giarratani, Frank & Pittsburgh (3) & 18.0 & 74 & 1.2 & 104 \\
\hline Goss, Ernst & Creighton (4) & 37.3 & 18 & 2.8 & 19 \\
\hline Grady, Stephen T. & Oak Ridge Nat Lab (2) & 22.0 & 45 & 2.0 & 31 \\
\hline Greenwood, Michael J. & Colorado (3) & 22.7 & 42 & 1.8 & 53 \\
\hline Guimaries, Paulo & Universidad Do Porto (2) & 10.3 & 113 & 0.8 & 131 \\
\hline Gunther, William D. & Alabama (2) & 19.0 & 68 & 2.0 & 31 \\
\hline Hall, William W., Jr. & UNC-Wilmington (2) & 5.5 & 155 & 1.0 & 109 \\
\hline Hansen, Niles M. & Texas (8) & 90.0 & 1 & 7.5 & 2 \\
\hline Harris, Thomas R. & Nevada Reno (6) & 41.3 & 14 & 2.1 & 30 \\
\hline Hastings, Stevens E. & Delaware (2) & 7.0 & 142 & 0.8 & 131 \\
\hline Haynes, Kingsley E. & George Mason (4) & 19.2 & 67 & 1.8 & 53 \\
\hline Hefner, Frank L. & South Carolina (2) & 13.0 & 95 & 0.8 & 131 \\
\hline Henry, Mark S. & Clemson (5) & 22.0 & 45 & 3.1 & 13 \\
\hline Herr, William M. & Southern Illinois (2) & 9.0 & 126 & 1.0 & 109 \\
\hline Herzog, Henry W., Jr. & Tennessee (6) & 26.2 & 34 & 1.8 & 53 \\
\hline Hite, James C. & Clemson (4) & 40.3 & 15 & 2.8 & 19 \\
\hline Houston, David B. & Pittsburgh (3) & 56.0 & 6 & 3.0 & 14 \\
\hline Hsing, Yu & SE Louisiana State (2) & 17.0 & 80 & 1.5 & 61 \\
\hline Hunt, Timothy L. & Upjohn Institute (2) & 8.0 & 133 & 1.5 & 61 \\
\hline Iden, George & American (2) & 12.0 & 100 & 2.0 & 31 \\
\hline Johnson, Thomas G. & Missouri (7) & 44.8 & 12 & 4.3 & 7 \\
\hline Jud, G. Donald & UNC-Greensboro (2) & 6.5 & 148 & 1.5 & 61 \\
\hline Katzman, Marvin T. & Texas-Dallas (2) & 24.0 & 37 & 2.0 & 31 \\
\hline Kau, James B. & Georgia (3) & 11.0 & 109 & 1.5 & 61 \\
\hline Knaap, Gerrit J. & Illinois (2) & 12.0 & 100 & 1.5 & 61 \\
\hline Knapp, Thomas A. & Penn State (3) & 24.5 & 36 & 1.5 & 61 \\
\hline Knox, Hugh W. & U.S. Dept. Commerce (2) & 6.0 & 149 & 2.0 & 31 \\
\hline Kort, John R. & U.S. Dept. Commerce (2) & 20.5 & 60 & 1.5 & 61 \\
\hline Kraft, Arthur & Ohio (2) & 12.0 & 100 & 1.5 & 61 \\
\hline Kraft, John & Florida (2) & 9.0 & 126 & 1.0 & 109 \\
\hline Kuehn, John A. & Missouri (3) & 12.0 & 100 & 1.8 & 53 \\
\hline Kurre, James A. & Penn St Erie (2) & 22.0 & 45 & 0.7 & 153 \\
\hline Laird, William E. & Florida State (2) & 8.5 & 130 & 1.5 & 61 \\
\hline Latham, William R. III & Delaware (5) & 33.0 & 23 & 3.5 & 11 \\
\hline Leathers, Charles G. & Alabama (2) & 14.0 & 91 & 1.5 & 61 \\
\hline Levernier, William B. & Georgia Southern (2) & 23.3 & 38 & 1.3 & 93 \\
\hline Liner, Gaines H. & UNC-Charlotte (2) & 37.0 & 19 & 2.0 & 31 \\
\hline Liu, Ben-Chieh & Oklahoma (2) & 13.0 & 95 & 1.0 & 109 \\
\hline Loviscek, Anthony L. & Indiana U Fort Wayne (2) & 20.0 & 62 & 1.5 & 61 \\
\hline Madsen, Ronald A. & UNC-Charlotte (2) & 9.5 & 121 & 1.0 & 109 \\
\hline Malizia, Emil E. & North Carolina (3) & 20.0 & 62 & 2.5 & 25 \\
\hline Marcouiller, David W. & Wisconsin (2) & 19.3 & 66 & 0.8 & 131 \\
\hline Martin, Randoph C. & South Carolina (3) & 38.0 & 17 & 2.5 & 25 \\
\hline Matson, Roger A. & Wyoming (2) & 9.3 & 123 & 0.8 & 131 \\
\hline Maxwell, Philip & Deakin U (2) & 9.0 & 126 & 1.0 & 109 \\
\hline Mazek, Warren F. & U.S. Coast Guard Acad (2) & 11.5 & 106 & 1.0 & 109 \\
\hline
\end{tabular}




\section{TABLE 11 (continued)}

Pages and Papers Published in The Review of Regional Studies by Author and Institution

\begin{tabular}{|c|c|c|c|c|c|}
\hline Author & Institution & $\begin{array}{l}\text { Adjusted } \\
\text { Pages }\end{array}$ & Rank & $\begin{array}{c}\text { Adjusted } \\
\text { Papers }\end{array}$ & Rank \\
\hline McDougall, Gerald S. & Wichita State (2) & 19.0 & 68 & 1.5 & 61 \\
\hline McKee, David L. & Kent State (2) & 6.0 & 149 & 2.0 & 31 \\
\hline McNamara, Kevin T. & Purdue (2) & 8.3 & 132 & 0.8 & 131 \\
\hline Meadows, Tommy C. & Austin Peay (2) & 8.5 & 130 & 1.5 & 61 \\
\hline Miernyk, William H. & West Virginia (3) & 19.0 & 68 & 3.0 & 14 \\
\hline Mikesell, John L. & Indiana (3) & 18.0 & 74 & 2.0 & 31 \\
\hline Miley, Harry W., Jr. & South Carolina (2) & 6.0 & 149 & 1.0 & 109 \\
\hline Miller, Edward M. & Rice & 55.0 & 7 & 4.0 & 8 \\
\hline Miller, James P. & U.S. Dept. Agric.(2) & 19.0 & 68 & 1.5 & 61 \\
\hline Mirakhor, Abbas & Alabama A\&M (2) & 7.5 & 140 & 1.0 & 109 \\
\hline Moody, Carlisle E., Jr. & William and Mary (2) & 25.0 & 35 & 2.0 & 31 \\
\hline Moriarty, Barry M. & North Carolina (3) & 48.0 & 10 & 3.0 & 14 \\
\hline Mulkey, W. David & Florida (8) & 45.3 & 11 & 4.7 & 5 \\
\hline Narayanan, Rangesan & Nevada Reno (2) & 7.0 & 142 & 0.5 & 156 \\
\hline Nicholas, James C. & Florida Atlantic (2) & 10.3 & 113 & 1.3 & 93 \\
\hline Niemi, Albert W., Jr. & Georgia (2) & 10.0 & 116 & 2.0 & 31 \\
\hline Nissan, Edward & Southern Miss (5) & 30.5 & 25 & 3.5 & 11 \\
\hline Olsen, Richard J. & Tennessee (2) & 15.5 & 85 & 1.5 & 61 \\
\hline Ostrosky, Anthony L. & Illinois State (2) & 7.0 & 142 & 2.0 & 31 \\
\hline Partridge, Mark D. & St. Cloud State (5) & 61.3 & 5 & 2.8 & 19 \\
\hline Persky, Joseph & Illinois-Chicago (3) & 20.2 & 61 & 1.3 & 93 \\
\hline Premus, Robert & Wright State (3) & 19.0 & 68 & 2.0 & 31 \\
\hline Pulver, Glen C. & Wisconsin (5) & 28.2 & 29 & 2.7 & 24 \\
\hline Raffie, Kambiz & Nevada Reno (2) & 10.2 & 115 & 0.6 & 155 \\
\hline Rasmussen, David W. & Florida State (X) & 26.5 & 33 & 4 & 8 \\
\hline Redman, Milton & U.S. Dept. Agric. (2) & 20.7 & 59 & 1.3 & 93 \\
\hline Renas, Stephen M. & Wright State (2) & 6.0 & 149 & 1.5 & 61 \\
\hline Rickman, Dan S. & Oklahoma State (4) & 39.3 & 16 & 1.8 & 53 \\
\hline Riefler, Roger F. & Nebraska (2) & 27.0 & 31 & 2.0 & 31 \\
\hline Rogers, Glenn & Wisconsin (2) & 7.7 & 139 & 0.7 & 153 \\
\hline Schaffer, William & Georgia Tech (2) & 8.0 & 133 & 2.0 & 31 \\
\hline Schlottmann, Alan M. & Tennessee (4) & 12.7 & 99 & 1.3 & 93 \\
\hline Schoening, Niles C. & Alabama Huntsville (2) & 8.0 & 133 & 1.0 & 109 \\
\hline Schreiner, Dean F. & Oklahoma State (2) & 10.8 & 111 & 0.8 & 131 \\
\hline Schulze, William D. & Cornell (2) & 16.7 & 81 & 0.8 & 131 \\
\hline Schwer, R. Keith & Nevada Las Vegas (3) & 17.3 & 78 & 1.3 & 93 \\
\hline Serow, William, J. & Florida State (9) & 71.3 & 2 & 6.8 & 3 \\
\hline Shaffer, Ron & Wisconsin (3) & 16.7 & 81 & 1.2 & 104 \\
\hline Shields, Martin & Penn State (3) & 21.8 & 52 & 1.2 & 104 \\
\hline Shonkwiler, J.S. & Nevada Reno (3) & 29.0 & 26 & 1.8 & 53 \\
\hline Siegel, Paul B. & Tel Hai College (3) & 22.3 & 44 & 1.3 & 93 \\
\hline Silberman, Jonathan & Arizona State West (2) & 13.5 & 93 & 1 & 109 \\
\hline Sirmans, C. F. & Connecticut (4) & 21.3 & 55 & 2.3 & 28 \\
\hline Smith, Dean $\mathrm{H}$. & Northern Arizona 2) & 23.0 & 39 & 1.5 & 61 \\
\hline Smith, W. C. & TVA (2) & 9.3 & 123 & 0.8 & 131 \\
\hline Spar, Michael A. & Florida State (3) & 17.3 & 78 & 1.3 & 93 \\
\hline Stephenson, Stan & Penn State (2) & 19.5 & 65 & 1.5 & 61 \\
\hline Stronge, William & Florida Atlantic (2) & 22.0 & 45 & 1.5 & 61 \\
\hline Sweeney, Larry E. & Ball State (2) & 8.0 & 133 & 1 & 109 \\
\hline Tam, Mo-Yin & Illinois Chicago (2) & 13.7 & 92 & 0.8 & 131 \\
\hline Uri, Noel D. & U.S. Dept. Energy (4) & 50.0 & 9 & 4 & 8 \\
\hline Weinstein, Robert & Bradley (2) & 9.0 & 126 & 1 & 109 \\
\hline Wells, Gary J. & Clemson (2) & 7.8 & 138 & 0.8 & 131 \\
\hline
\end{tabular}


TABLE 11 (continued)

Pages and Papers Published in The Review of Regional Studies by Author and Institution

\begin{tabular}{llcccr}
\hline Author & Institution & $\begin{array}{c}\text { Adjusted } \\
\text { Pages }\end{array}$ & Rank & $\begin{array}{c}\text { Adjusted } \\
\text { Papers }\end{array}$ & Rank \\
\hline West, Carol T. & Florida (2) & 21.5 & 54 & 1.5 & 61 \\
White, Fred C. & Georgia (2) & 11.5 & 106 & 1 & 109 \\
White, Michael J & Brown 2) & 35.0 & 21 & 1.5 & 61 \\
Yu, Eden S. H. & Oklahoma (2) & 13.0 & 95 & 1 & 109 \\
Ziegler, Joseph A. & Arkansas (2) & 17.5 & 77 & 1.5 & 61 \\
\hline
\end{tabular}

Source: The Review of Regional Studies, Volume 3, Number 1, through Volume 29, Number 2.

\section{SUMMARY AND CONCLUSIONS}

In this paper we have looked at some characteristics of the Southern Regional Science Association and its publication, The Review of Regional Studies. We find that support for our organization comes primarily from southern states, not at all unexpectedly, and from the District of Columbia. Outside the Deep South, significant support comes from Texas, Oklahoma, Virginia, West Virginia, Pennsylvania, and Illinois, with persons from all states and many foreign countries also making important contributions.

\section{REFERENCES}

Benson, Oliver, and Charles M. Bonjean. "The Social Science Quarterly, 1920-1970; A Case Study of Organizational Growth." Social Science Quarterly 50 (1970), 806-825.

Carnegie Foundation for the Advancement of Teaching. A Classification of Institutions of Higher Learning. Princeton, NJ: Carnegie Foundation, 1987.

Durden, Garey C. "An Analysis of Publishing Performance in Social Science Quarterly." Social Science Quarterly 72 (1991), 181-188.

Durden, Garey C., and James Marlin. "An Analysis of Contributions and Contributors to Public Choice and the Journal of Law and Economics." Public Choice 65 (1990), 101-141.

Kau, James, and Linda Johnson. "Regional Science Programs: A Ranking Based on Publication Performance." Journal of Regional Science 23 (1983), 177-186.

Marlin, James W., and Garey C. Durden. "An Analysis of Contributions and Contributors in Economic Education Research." Journal of Economic Education 24 (1993), 176-186.

Shogren, Jason F., and Garey C. Durden. "The First Fifteen Years: Contributors and Contributions to the Journal of Environmental Economics and Management, 1974-1988." Journal of Environmental Economics and Management 20 (1991), 205-209. 